\title{
The effect of a virtual ward program on emergency services utilization and quality of life in frail elderly patients after discharge: a pilot study
}

\author{
Doris Y P Leung' \\ Diana Tze-Fan Lee' \\ Iris F K Lee' \\ Lai-Wah Lam' \\ Susanna WY Lee ${ }^{2}$ \\ May W M Chan ${ }^{3}$ \\ Yin-Ming Lam ${ }^{4}$ \\ Siu-Hung Leung ${ }^{5}$ \\ Pui-Chi Chiu ${ }^{6}$ \\ Nelly K F Ho ${ }^{7}$ \\ Ming-Fai IP ${ }^{8}$ \\ May MY Hui ${ }^{8}$
}

'The Nethersole School of Nursing, The Chinese University of Hong Kong, Shatin, Hong Kong; ${ }^{2}$ Hospital Authority Head Office, ${ }^{3}$ Kowloon West Cluster, ${ }^{4} \mathrm{New}$ Territories West Cluster, ${ }^{5}$ Kowloon East Cluster. ${ }^{6}$ United Christian Hospital, ${ }^{7}$ Kowloon Hospital, ${ }^{8}$ Tuen Mun Hospital, Hong Kong Hospital Authority, Kowloon, Hong Kong

This article was published in the following Dove Press journal:

Clinical Interventions in Aging

3 February 2015

Number of times this article has been viewed

Introduction: Attendance at emergency departments and unplanned hospital readmissions are common for frail older patients after discharge from hospitals. A virtual ward service was piloted to deliver "hospital-at-home" services by community nurses and geriatricians to frail older patients immediately after their discharge from hospital to reduce emergency services utilization.

Objectives: This study examined the impacts of the virtual ward service on changes in the patients' emergency attendance and medical readmissions, and their quality of life (QOL).

Methods: A matched-control quasi-experimental study was conducted at four hospitals, with three providing the virtual ward service (intervention) and one providing the usual community nursing care (control). Subjects in the intervention group were those who are at high risk of readmission and who are supported by home carers recruited from the three hospitals providing the virtual ward service. Matched control patients were those recruited from the hospital providing usual care. Outcome measures include emergency attendance and medical readmission in the past 90 days as identified from medical records, and patient-reported QOL as measured by the modified Quality-of-Life Concerns in the End of Life Questionnaire (Chinese version). Wilcoxon signed-rank tests compared the changes in the outcome variables between groups.

Results: A total of 39 patients in each of the two groups were recruited. The virtual ward group showed a greater significant reduction in the number of unplanned emergency hospital readmissions $(-1.41 \pm 1.23$ versus $-0.77 \pm 1.31 ; P=0.049)$ and a significant improvement in their overall QOL ( $\mathrm{n}=18 ; 0.60 \pm 0.56$ versus $0.07 \pm 0.56 ; P=0.02$ ), but there was no significant difference in the number of emergency attendances $(-1.51 \pm 1.25$ versus $-1.08 \pm 1.48 ; P=0.29)$.

Conclusion: The study results support the effectiveness of the virtual ward service in reducing unplanned emergency medical readmissions and in improving the QOL in frail older patients after discharge.

Keywords: elderly, emergency attendance, emergency medical readmission, emergency services utilization, quality of life, virtual ward

\section{Introduction}

Attendance at emergency departments and unplanned hospital readmissions are common for frail older patients after discharge from the hospital. ${ }^{1}$ Patients with chronic illnesses frequently perceived powerlessness in managing their disease after hospital discharge, and they are prompted to seek hospital readmission immediately upon symptom exacerbation. ${ }^{2}$ Unplanned readmission is usually defined as readmission to the hospital within 28 days postdischarge..$^{3,4}$

In Hong Kong, it was estimated that the unplanned readmission rate was $16.7 \%$ in the general population, ${ }^{3}$ and more than $20 \%$ in the elderly subpopulation. ${ }^{4}$ The Hong Kong

\footnotetext{
Correspondence: Diana Tze-Fan Lee The Nethersole School of Nursing, The Chinese University of Hong Kong, 7/F, Esther Lee Building, The Chinese University of Hong Kong, Shatin, Hong Kong

Tel +85239436227

Fax +852 29942107

Email tzefanlee@cuhk.edu.hk
} 
Hospital Authority has developed a 14-variable predictive model - the Hospital Administration Risk Reduction Programme for the Elderly (HARRPE) - to predict the risk of emergency medical admissions in 28 days after an index hospital visit among elderly patients aged 65 years or above. ${ }^{4}$ An elderly patient with a HARRPE score of $\geq 0.2058$ was considered as having a high risk of emergency admission.

According to the unpublished statistical record from the medical unit of six hospitals under the operation of the Hospital Authority in 2009, the average length of stay for patients with a HARRPE score of $\geq 0.4$ is 23 days, which is much higher than the corresponding figure of 5.9 days in the Hong Kong general population. ${ }^{3}$ Currently, patients are provided with a community nursing service after hospital discharge in Hong Kong; it is, therefore, essential to develop effective intervention programs to help prevent unplanned readmission among those elderly patients who are at high risk.

The concept of virtual ward is a new model of care that was pioneered in 2004 in the UK. ${ }^{5,6}$ The aim is to provide clients at high risk of hospital readmission with intensive multidisciplinary and coordinated extensive services so as to facilitate their receiving the necessary care in their own homes, thereby reducing hospital readmissions. Since the concept of virtual ward employs the systems, staffing, and daily routine of a hospital ward to deliver care to clients in their home settings, ${ }^{6}$ the virtual ward does not have a physical ward building. Patients admitted to the virtual ward are visited by community nurses for the delivery of routine nursing care normally provided in the hospital, such as measuring their blood sugar levels, administering insulin, and performing wound dressing, at their homes. Physicians and other allied health care professionals may also be involved, depending on the patient's health condition. ${ }^{5}$

Evidence supporting the effectiveness of the virtual ward service was reported in the literature. The hospital-at-home care resulted in a reduction in the length of hospitalization compared to inpatient care in a systematic review, ${ }^{7}$ and it was shown to have a better effect in reducing emergency visits in comparison with usual home care visits in patients with acute or complex conditions requiring care for an anticipated 5-10 days. $^{8}$ A 7-year longitudinal study on a large cohort of patients receiving acute hospital services at home reported low rates of unexpected mortality $(0.15 \%)$ and unplanned returns to hospital $(4.2 \%) .{ }^{9}$ Previous studies also supported that patient satisfaction was greater with virtual ward care, ${ }^{6,8}$ and potential improvements in quality of life (QOL), depression, and nutritional status in elderly patients with chronic heart failure have also been reported. ${ }^{10}$ The greater satisfaction might be attributed to a more personal style of care and a feeling that staying at home was therapeutic. ${ }^{11}$ The purpose of this study is to evaluate the effectiveness of the virtual ward service as compared to community nursing care on health services utilization and the QOL of patients who are at extremely high risk for emergency readmission.

\section{Materials and methods \\ Subjects}

The study was conducted from March 2012-January 2013 at the Kowloon and New Territories West Clusters under the catchment areas of community nursing services of four regional hospitals of the Hong Kong Hospital Authority. Subjects were eligible if they had been discharged from hospital, had a HARRPE score $\geq 0.4$, or had a major functional disability as clinically indicated, and were being supported by home carers who were living with the patients. Subjects were excluded if they were psychologically or physically unable to communicate. The study was approved by the Institutional Review Board of Kowloon East Cluster, Kowloon Central Cluster, Kowloon West Cluster, and New Territories West Cluster of Hong Kong Hospital Authority. Informed consent was obtained from all subjects before their participation.

This is a matched-control quasi-experimental study. A total of 40 pairs of patients and their carers recruiting from three of the four participating hospitals were assigned to the intervention group, while another 40 matched patient-carer dyads recruiting from the remaining hospital were assigned to the control group. Five criteria were used to select the patient-carer dyads in the control group; these included: 1) disease type of the patient; 2) frailty level of the patient as measured by the validated Clinical Frailty Index; ${ }^{12,13} 3$ ) sex of the patient; 4 ) age of the patient ( \pm 5 years); and 5) the carer's relationship with the patient (maid/spouse/children/relatives). This sample size is considered to be adequate for estimating a between-group difference for the pilot study. ${ }^{14}$

Eligible patients were admitted to the three selected hospitals delivering the virtual ward service and their carers were invited to participate in the study. The screening of the subjects was performed by virtual ward nurses at the participating hospitals. The research assistant of the project then conducted the baseline assessment to both the patient and the carer in the intervention group at home before the implementation of the service by the virtual ward team. For each consented patient-carer dyad in the intervention group, a matched control dyad was then selected from the fourth hospital by using the 
above five matching criteria by the nurses in the hospital. The research assistant then administered the baseline assessment to the consented matched control at their homes.

Participants in the intervention group received the virtual ward service while those in the control group received the usual community nursing service provided to patients discharged from hospitals in Hong Kong. At 3 months or discharge from the virtual ward (whichever is earlier), a follow-up survey using a standardized questionnaire via faceto-face interviews was administered to both the patients and their carers. An outline of the study is shown in Figure 1.

\section{Measures}

Five types of hospital services utilization were retrieved from the patients' medical records. Three measures that are related to services utilization of the emergency department were reported in the current study: length of the hospitalization via emergency department; number of unplanned emergency hospital admissions; and number of emergency attendances in the past 90 days.

The patient's QOL was measured by the locally validated instrument, namely, the Chinese version modified Quality-of-Life Concerns in the End of Life Questionnaire (mQOLC-E). ${ }^{15,16}$ The scale consists of 23 items measuring the six dimensions of: 1) negative emotions; 2) physical discomfort; 3) value of life; 4) existential distress; 5) care and support; and 6) food-related concerns, on a 4-point Likert scale, and the scores ranged from 1, "the least satisfaction"

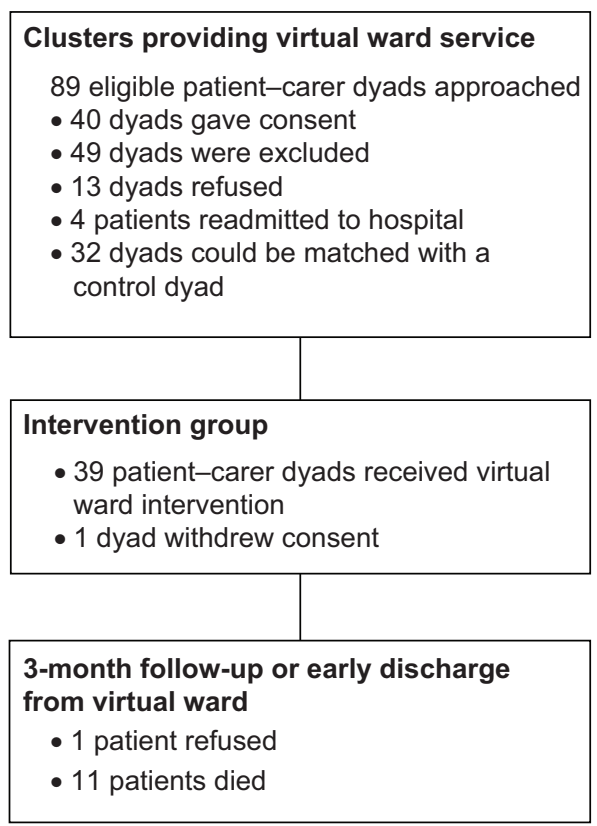

to 4, "the most satisfaction" toward the condition, with higher scores indicating a better QOL.

Modifications were made to two items on the care and support concern subscale of the instrument regarding perception of the care the patient received. In particular, the two items originally asking the respondents to rate the care they received in hospital have been changed to the care they are currently receiving (ie, the virtual ward service for subjects in the intervention group and the usual community nursing care in the control group). Aggregate scores were created for the overall score and each of the six dimensions of the scale by averaging the corresponding items. Cronbach's alpha value for the overall score was 0.835 , and those for the six dimensions ranged from 0.791 for value of life to 0.879 for negative emotions in the current sample.

\section{Virtual ward program}

The patients in the intervention group received the service provided by the virtual ward health care team that consisted of nurses and physicians in the respective cluster. The virtual ward service focused on the provision of hospital-level care to patients and health care supports to their carers at their homes. Patients were then individually assigned to a virtual ward nurse (primary nurse); and the virtual ward nurse conducted the first home visit as soon as possible within 48 hours after the patient was discharged from the hospital. The virtual ward physician might conduct a physician home visit within the first week after the patient was discharged.

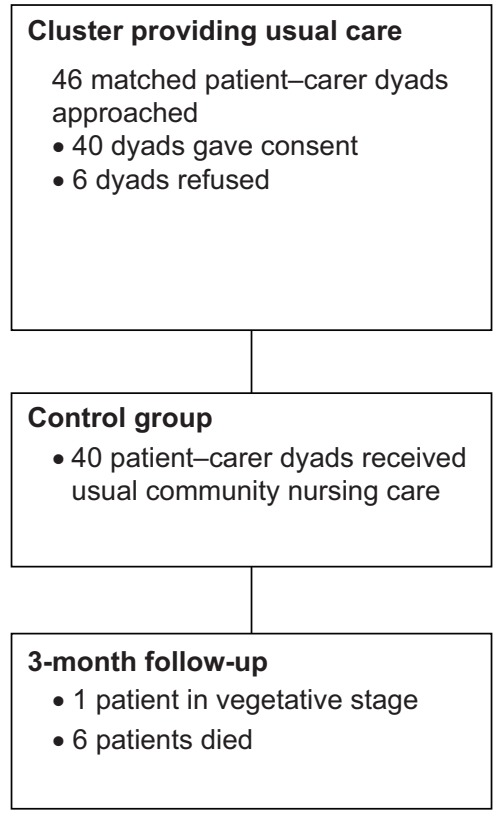

Figure I Flow chart of subjects through the study. 
The focus of the first home visit was mainly on health assessment. Based on the health assessment results, the virtual ward nurses planned the schedule of home visits which could range from daily to once every 2 weeks. During the home visits, the virtual ward nurses provided direct patient care and health education, such as monitoring and handling symptom exacerbation, to the patients and their carers. The virtual ward nurses also provided psychosocial supports to both the patients and their carers. The schedule of home visits was individualized, and each patient was visited about four times per week on average during the study. In addition, an extended service beyond office hours and a hotline consultation service were also provided for the patients and their carers by the virtual ward teams. This additional service aims to enable the patients to have fast-track medical consultations by establishing various fast-track services, such as fast-track clinic, direct clinical admission, and enhanced nonemergency ambulance transportation service support. The frequency of calls to the hotline was reported to be at most three times in a week during the study.

Patients in the control group received community nursing service, referred by the physician in charge, that was based on the patients' specific nursing care needs postdischarge; this included wound dressing, catheterization, and health education for the patients' self-care of their chronic diseases. The case would be closed if those specific health problems were resolved.

\section{Statistical analysis}

Descriptive statistics, including frequencies and percentages for categorical data and means and standard deviations for continuous data, were calculated for the baseline data of the sample. Normality of data was checked by using $z$-tests for skewness and kurtosis. ${ }^{17}$ Paired $t$-tests for normal data and Wilcoxon signed-rank tests for nonnormal data were used to compare the differences in the changes of the outcome variables between the two groups using all available data. We also conducted a sensitivity analysis for hospital service utilization by excluding those patients who had died during the study. All statistical tests were two-sided, and a $P$-value $<0.05$ was considered statistically significant. All the analyses were performed by using SPSS version 20.0 (IBM Corporation, Armonk, NY, USA).

\section{Results}

A total of 89 eligible patient-carer dyads in the intervention group and 46 dyads in the control group were invited to participate in the study. For the intervention group, 13 dyads refused, patients in four dyads were readmitted to the hospital, and 32 dyads were excluded because they were unable to be matched with a control dyad. For the control group, six dyads refused to join the study. One dyad in the intervention group withdrew their consents before receiving the virtual ward service, because the patient was expecting to frequently travel to the People's Republic of China in the near future and, therefore, could not receive the service. At 3 months, 27 patients in the intervention group and 33 patients in the control group were successfully followed-up. Among patients who were lost to follow-up, eleven died and one refused to complete the questionnaire in the intervention group, and six died and one was in a vegetative stage in the control group.

Thus, a total of 39 matched patient pairs in the two groups were available for the analysis on hospital services utilization. For the QOL, due to loss to follow-up and missing data at the item level, analyses were performed based on complete cases. The demographics of the participants and their carers are shown in Table 1.

Table I Demographic characteristics of patients and carers by group $(\mathrm{N}=39)$

\begin{tabular}{|c|c|c|}
\hline Characteristics & $\begin{array}{l}\text { Intervention } \\
(n=39)\end{array}$ & $\begin{array}{l}\text { Control } \\
(n=39)\end{array}$ \\
\hline \multicolumn{3}{|l|}{ Patients } \\
\hline Age, mean $\pm S D$ & $80.2 \pm 7.7$ & $80.5 \pm 8.5$ \\
\hline Clinical Frailty Index, mean \pm SD & $6.64 \pm 1.35$ & $6.15 \pm 1.44$ \\
\hline Male, n (\%) & $28(71.8)$ & $29(74.4)$ \\
\hline \multicolumn{3}{|l|}{ Educational level, n (\%) } \\
\hline Not educated/kindergarten & $19(48.7)$ & $16(4 \mid .0)$ \\
\hline Primary school & $17(43.6)$ & $14(35.9)$ \\
\hline Above primary school & $3(7.7)$ & $9(23.1)$ \\
\hline \multicolumn{3}{|l|}{ Diagnosis of disease, $\mathbf{n}(\%)$} \\
\hline COPD & $12(30.8)$ & $12(30.8)$ \\
\hline Chronic heart failure & $10(25.6)$ & $10(25.6)$ \\
\hline Cancer & 7 (17.9) & $7(17.9)$ \\
\hline Others & $10(25.6)$ & $10(25.6)$ \\
\hline \multicolumn{3}{|l|}{ Carer's relationship with } \\
\hline \multicolumn{3}{|l|}{ patient, n (\%) } \\
\hline Spouse & $19(48.7)$ & $22(56.4)$ \\
\hline Child & $16(41.0)$ & $15(38.5)$ \\
\hline Relatives & $2(5.1)$ & $0(0)$ \\
\hline Maid & $2(5.1)$ & $2(5.1)$ \\
\hline \multicolumn{3}{|l|}{ Carers } \\
\hline Age, mean $\pm S D$ & $58.7 \pm 17.9$ & $58.7 \pm 17.0$ \\
\hline Male, n (\%) & $9(23.1)$ & $5(12.8)$ \\
\hline \multicolumn{3}{|l|}{ Educational level, n (\%) } \\
\hline Not educated/kindergarten & $12(30.8)$ & $8(20.5)$ \\
\hline Primary school & $7(17.9)$ & II (28.2) \\
\hline Above primary school & $20(5 \mid .3)$ & $20(5 \mid .3)$ \\
\hline
\end{tabular}

Note: Percentages may not sum up to $100 \%$ due to rounding-off.

Abbreviations: SD, standard deviation; COPD, chronic obstructive pulmonary disease. 
Table 2 Comparison of changes in hospital services utilization from baseline to follow-up of matched patient pairs in the two groups

\begin{tabular}{|c|c|c|c|c|c|c|c|c|}
\hline \multirow[t]{2}{*}{ Hospital service } & \multirow{2}{*}{$\begin{array}{l}\text { Matched } \\
\text { pair }\end{array}$} & \multicolumn{3}{|c|}{ Intervention } & \multicolumn{3}{|l|}{ Control } & \multirow[t]{2}{*}{$P$} \\
\hline & & Baseline & Follow-up & Change & Baseline & Follow-up & Change & \\
\hline $\begin{array}{l}\text { Length of hospitalization } \\
\text { via emergency admission } \\
\text { in past } 90 \text { days }\end{array}$ & 39 & $15.03 \pm 17.52$ & $3.4 I \pm 8.09$ & $-11.62 \pm 17.91$ & $14.28 \pm 17.68$ & $9.43 \pm 18.74$ & $-4.38 \pm 26.4 I$ & 0.14 \\
\hline $\begin{array}{l}\text { Number of admissions via } \\
\text { emergency admission in } \\
\text { past } 90 \text { days }\end{array}$ & 39 & $1.97 \pm 1.31$ & $0.56 \pm 0.72$ & $-1.4 I \pm 1.23$ & $1.68 \pm 1.23$ & $0.90 \pm 1.08$ & $-0.77 \pm 1.31$ & $0.049 *$ \\
\hline $\begin{array}{l}\text { Number of A\&E attendance } \\
\text { in past } 90 \text { days }\end{array}$ & 39 & $2.15 \pm 1.27$ & $0.64 \pm 0.78$ & $-1.5 \mid \pm 1.25$ & $2.08 \pm 1.5 \mid$ & $1.00 \pm 1.09$ & $-1.08 \pm 1.48$ & 0.29 \\
\hline
\end{tabular}

Notes: Data are reported with mean and standard deviation. Wilcoxon signed-rank sum tests were used. $* P$-value $<0.05$.

Abbreviation: A\&E, accident and emergency.

The results reported in Tables 2 and 3 revealed that the intervention group showed a significantly greater reduction in the number of unplanned emergency hospital admissions $(-1.41 \pm 1.23$ versus $-0.77 \pm 1.31 ; P=0.049)$. Although there were no significant differences in both the length of hospitalization via emergency admission $(-11.62 \pm 17.91$ versus $-4.38 \pm 26.41 ; P=0.14)$ and the number of emergency attendances $(-1.51 \pm 1.25$ versus $-1.08 \pm 1.48 ; P=0.29)$ in the past 90 days between the two groups, the decreases in these two clinical measures were greater in the intervention group (Table 2). Sensitivity analysis by excluding those patients who had died during the study period also gives similar results on the three clinical outcomes (Table 3).

For QOL, patients receiving the virtual ward service reported higher mean values in the changes of the overall scores and the scores in all the six dimensions of mQOLC-E from the baseline to the follow-up than patients receiving the community nursing service (Table 4 ). Significant differences were observed in the overall QOL scores $(0.60 \pm 0.56$ versus $0.07 \pm 0.56 ; P=0.02)$ and the three dimensions of foodrelated concerns $(0.82 \pm 0.87$ versus $-0.14 \pm 0.95 ; P=0.003)$, negative emotions $(0.73 \pm 0.74$ versus $0.02 \pm 1.03 ; P=0.01)$, and existential distress $(0.72 \pm 1.06$ versus $0.15 \pm 0.81$; $P=0.04)$.

\section{Discussion}

Our pilot study shows that for frail patients with chronic diseases, the virtual ward service, with physicians and nurses undertaking visits to the patient's home, results in a significantly greater reduction in the number of admissions via emergency admissions as compared to matched controls receiving the usual community nursing service. The result on frequency of admission via emergency admission remained significant in the sensitivity analysis by excluding those patients who died during the study period. Although nonsignificant, the reductions in length of stay via emergency admission and emergency attendance were also greater in the intervention group. Consistent with a previous study, our study also showed that health care provided at home by a multidisciplinary team involving physicians, nurses, and carers is effective in reducing emergency visits than is the usual care provided by community nurses and carers. ${ }^{8}$ Compared to a previous local randomized controlled trial (RCT) which examined the effectiveness of nursing home

Table 3 Comparison of changes in hospital services utilization from baseline to follow-up of matched patient pairs in the two groups excluding those who died

\begin{tabular}{|c|c|c|c|c|c|c|c|c|}
\hline \multirow[t]{2}{*}{ Hospital service } & \multirow{2}{*}{$\begin{array}{l}\text { Matched } \\
\text { pair }\end{array}$} & \multicolumn{3}{|l|}{ Intervention } & \multicolumn{3}{|l|}{ Control } & \multirow[t]{2}{*}{$P$} \\
\hline & & Baseline & Follow-up & Change & Baseline & Follow-up & Change & \\
\hline $\begin{array}{l}\text { Length of hospitalization } \\
\text { via emergency admission } \\
\text { in past } 90 \text { days }\end{array}$ & 24 & $|7.2| \pm 2 \mid .09$ & $4.67 \pm 9.97$ & $-12.54 \pm 2 \mid .74$ & $|1.50 \pm||.2|$ & 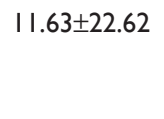 & $0.13 \pm 25.12$ & 0.12 \\
\hline $\begin{array}{l}\text { Number of admissions via } \\
\text { emergency admission in } \\
\text { past } 90 \text { days }\end{array}$ & 24 & $2.17 \pm 1.37$ & $0.63 \pm 0.77$ & $-1.54 \pm 1.22$ & $1.75 \pm 1.33$ & $1.08 \pm 1.25$ & $-0.67 \pm 1.49$ & $0.038^{*}$ \\
\hline $\begin{array}{l}\text { Number of } A \& E \text { attendance } \\
\text { in past } 90 \text { days }\end{array}$ & 24 & $2.33 \pm 1.37$ & $0.7 I \pm 0.86$ & $-1.63 \pm 1.24$ & $2.38 \pm 1.66$ & $1.13 \pm 1.23$ & $-1.25 \pm 1.59$ & 0.50 \\
\hline
\end{tabular}

Notes: Data are reported with mean and standard deviation. Wilcoxon signed-rank sum tests were used. $* P$-value $<0.05$.

Abbreviation: A\&E, accident and emergency. 
Table 4 Comparison of changes in QOL scores from baseline to follow-up of the matched patients in the two groups

\begin{tabular}{|c|c|c|c|c|c|c|c|c|}
\hline \multirow[t]{2}{*}{ mQOLC-E dimension } & \multirow{2}{*}{$\begin{array}{l}\text { Matched } \\
\text { pair }\end{array}$} & \multicolumn{3}{|c|}{ Intervention } & \multicolumn{3}{|l|}{ Control } & \multirow[t]{2}{*}{$P$} \\
\hline & & Baseline & Follow-up & Change & Baseline & Follow-up & Change & \\
\hline Overall mQOLC-E & 18 & $2.8 \mathrm{I} \pm 2.58$ & $3.42 \pm 0.60$ & $0.60 \pm 0.56$ & $2.78 \pm 0.72$ & $2.85 \pm 0.75$ & $0.07 \pm 0.56$ & $0.02^{\mathrm{a}, *}$ \\
\hline Physical discomfort & 22 & $2.49 \pm 0.67$ & $3.16 \pm 0.91$ & $0.67 \pm 0.92$ & $2.18 \pm 0.88$ & $2.39 \pm 1.05$ & $0.20 \pm 0.96$ & $0.17^{\mathrm{b}}$ \\
\hline Food-related concerns & 22 & $2.4 \mathrm{I} \pm 0.98$ & $3.23 \pm 0.84$ & $0.82 \pm 0.87$ & $2.52 \pm 0.96$ & $2.39 \pm 0.96$ & $-0.14 \pm 0.95$ & $0.003^{\mathrm{b}, * *}$ \\
\hline Care and support & 21 & $3.44 \pm 0.43$ & $3.87 \pm 0.27$ & $0.43 \pm 0.46$ & $3.19 \pm 0.63$ & $3.31 \pm 0.39$ & $0.12 \pm 0.59$ & $0.09^{a}$ \\
\hline Negative emotions & 20 & $2.83 \pm 0.87$ & $3.56 \pm 0.46$ & $0.73 \pm 0.74$ & $2.76 \pm 1.00$ & $2.78 \pm 1.06$ & $0.02 \pm 1.03$ & $0.0 I^{\mathrm{b}, * * *}$ \\
\hline Existential distress & 20 & $2.52 \pm 0.92$ & $3.23 \pm 0.92$ & $0.72 \pm 1.06$ & $2.62 \pm 0.94$ & $2.77 \pm 0.99$ & $0.15 \pm 0.81$ & $0.04^{\mathrm{b}, *}$ \\
\hline Value of life & 19 & $3.17 \pm 0.89$ & $3.39 \pm 0.78$ & $0.22 \pm 0.85$ & $2.91 \pm 0.88$ & $2.89 \pm 0.93$ & $-0.01 \pm 0.40$ & $0.23^{\mathrm{a}}$ \\
\hline
\end{tabular}

Notes: Data are reported with mean and standard deviation. ${ }^{a}$ Wilcoxon signed-rank sum tests; ${ }^{\text {bPaired }} t$-test. ${ }^{*} P$-value $<0.05$. ${ }^{* * P}$-value $<0.01 .{ }^{* * *} P$-value $<0.005$. Abbreviations: QOL, quality of life; mQOLC-E, modified Quality-of-Life Concerns in the End of Life Questionnaire.

visits driven by a protocol which used the Omaha scheme and the standard community nursing service, ${ }^{18}$ our subjects were older and frailer, and they had to be taken care of, which means that they are expected to be at a higher risk of emergency hospitalization.

Their study showed that home visits driven by a protocol that used the Omaha scheme had a similar effect as the basic community nursing service on the frequency of hospitalization. The current results highlight the potentially important role of the main feature of targeting those patients who are at high risk of emergency hospitalization in the virtual ward services, which distinguishes it from other programs designed to prevent hospitalization. Our study had provided new evidence for the efficacy of the virtual ward service, as compared to home visits by community nurses, in reducing the number of readmissions. This suggests that providing a more intensive care at home by a team of multidisciplinary health care professionals might be more effective than a low intervention dose, ie, home visits delivered by community nurses only for frail older patients. ${ }^{18,19}$ This observation also echoed the views that patients with chronic, functional, and relatively intractable health problems require a higher level of intervention..$^{20}$ However, further studies using an RCT with adequate power on testing the effectiveness of the virtual ward service on the target population are warranted.

The mortality rate in the intervention group was higher than that in the control group, although the difference was not significant (McNemar test, $P=0.27$ ). It is possible that some of the patients were at their end stage of life and that the patients as well as their carers might prefer to stay at home and forgo life-sustaining treatment. ${ }^{21}$ Although the accessibility of hospitals and the low cost for a hospital pay in Hong Kong might be important factors for unplanned readmission, ${ }^{16}$ both patients and their carers may not opt for this option unless necessary. Hospitalization inevitably induces stress both to patients and their family carers. ${ }^{22,23}$
Repeated emergency hospital admissions are also a significant and disruptive event for families, because it may herald the possibility of an uncertain future and anticipated loss to both the families and the patients. ${ }^{24}$ Thus, it is possible that the frail patients or their carers may feel that it is safer for the patients to stay in the hospital if their conditions are out of control even though both of them may not be well-prepared for such readmission. ${ }^{25}$

Furthermore, many forms of care and support, particularly comfort measures like feeding and bathing, that family members normally provide to older adults with multiple comorbidities or impaired functional ability are to be continued but in a hospital setting. ${ }^{24}$ This situation is especially true in Chinese society, because the Chinese have a strong sense of moral obligation to take care of a sick family member. ${ }^{26}$ Hence, it is very important to manage the patients' condition by providing timely professional advice, such as a change of medication prescription, so that some emergency admissions could be avoided. The close monitoring of the patients' health condition and the prompt adjustment of medication orders are risk factors of readmission ${ }^{27}$ that can be remedied by better health care coordination, such as that provided by the virtual ward service.

Patients receiving the virtual ward service reported greater improvements in the overall score and all the six dimension scores in QOL as compared to patients receiving the community nursing service; this is in line with the findings from a previous study on elderly patients with chronic heart failure. ${ }^{10}$ In particular, the changes in the overall QOL score and the three dimensions of food-related concerns, negative emotions, and existential distress were statistically significant between the two groups. The results might suggest that the virtual ward service may exert positive effects on the QOL of the patients by providing individualized care, health education, and emotional support to the patients and their carers so that the conditions of the patients can be better monitored. 
However, the results from our nonrandomized matched control study should be interpreted with caution because of potential bias due to patients' loss to follow-ups.

The study had several limitations that are worthy of note. First, this is a pilot study, and it may have produced imprecise estimates of the differences in the outcome variables between the groups. Thus, the results should be interpreted with caution. Nevertheless, our study has produced an estimate of the effect size of the virtual ward service to the usual community nursing service for future studies. Second, although we have successfully recruited matched controls by using the five criteria for comparison, it is still possible that we have not controlled other possible confounders, such as the characteristics of the carers and the study site, and this might have induced bias into the study results. However, we were not able to recruit control subjects in the experimental hospitals due to the time constraint of this pilot study, and the small sample size precludes the inclusion of the carers' characteristics in the analysis.

Further studies should consider using RCT with a larger sample size, to examine the effectiveness of the virtual ward service in the target population. Third, we have high attrition rates in patients in both groups, and this might have introduced bias in the results of QOL. Fourth, we have a mixture of disease-specific patient groups including chronic obstructive pulmonary disease (COPD), chronic heart failure, cancer, and other diseases in our study. Each patient group might require different types of interventions at home, and some of the required interventions might not be covered by the virtual ward service. Thus, it may be possible that the impact of the service might differ in accordance with the disease types of the patients.

Given the encouraging results obtained in this pilot study, it is worthwhile to further investigate the effect of the virtual ward service on patients with some specific diseases, such as COPD and chronic heart failure, who are at high risk of emergency hospitalization individually. Finally, we did not collect cost information of the virtual ward and the usual community nursing service for this pilot study due to time and financial constraints. Because the virtual ward service is still available at the three experimental hospitals, one of the main focuses of further studies should be on the costeffectiveness of the service as compared to the community nursing care.

\section{Conclusion}

Supported by the results of this pilot study, the virtual ward service is effective in reducing unplanned hospital readmissions and improving the patients' QOL. It is also potentially effective in reducing the patients' length of stay via emergency admissions and emergency attendances. These results support the potential benefits of virtual ward service for frail elderly patients who live with their carers.

\section{Acknowledgment}

This study was supported by grant (177067) from the Hong Kong Hospital Authority, Hong Kong. We would like to thank Mr Pui-On Li for his contribution in study design and data collection of the study.

\section{Disclosure}

The authors report no conflicts of interest in this work.

\section{References}

1. NSW Department of Health. Transitional Aged Care. North Sydney: NSW Department of Health; 2006. Available from: http://www.archi. net.au/documents/resources/models/transitional_care/transitionalagedcare.pdf. Accessed September 19, 2014.

2. Yu DS, Lee DT, Woo J. The revolving door syndrome: the Chinese COPD patients' perspectives. J Clin Nurs. 2007;16(9):1758-1760.

3. Wong EL, Cheung AW, Leung MC, et al. Unplanned readmission rates, length of hospital stay, mortality, and medical costs of ten common medical conditions: a retrospective analysis of Hong Kong hospital data. BMC Health Serv Res. 2011;11:149.

4. Tsui E, Au S, Wong C, Cheung A, Lam P. Development of an automated model to predict the risk of elderly emergency medical admissions within a month following an index hospital visit: A Hong Kong Experience. Health Informatics J. Epub 2013 Dec 18.

5. Lewis G. Virtual wards: real nursing. Nurs Stand. 2007;21(43):64.

6. Lewis G. Predictive Modeling in Action: How 'Virtual Wards' Help High-Risk Patients Receive Hospital Care at Home. Issues in International Health Policy. 2010;94:1-16.

7. Shepperd S, Iliffe S. Hospital at home versus in-patient hospital care [review]. Cochrane Database Syst Rev. 2005;3:CD000356.

8. Stewart M, Sangster JF, Ryan BL, et al. Integrating Physician Services in the Home: evaluation of an innovative program. Can Fam Physician. 2010;56(11):1166-1174.

9. Montalto M, Lui B, Mullins A, Woodmason K. Medically-managed Hospital in the Home: 7 year study of mortality and unplanned interruption. Aust Health Rev. 2010;34(3):269-275.

10. Tibaldi V, Isaia G, Scarafiotti C, et al. Hospital at home for elderly patients with acute decompensation of chronic heart failure: a prospective randomized controlled trial. Arch Intern Med. 2009;169(17):1569-1575.

11. Wilson A, Wynn A, Parker H. Patient and carer satisfaction with 'hospital at home': quantitative and qualitative results from a randomised controlled trial. Br J Gen Pract. 2002;52(474):9-13.

12. Geriatric Medicine Research Unit, Dalhousie University [homepage on the Internet]. Clinical Frailty Scale; 2012 [updated January 14, 2011 ; cited December 6, 2012]. Available from: http://geriatricresearch.medicine. dal.ca/clinical_frailty_scale.htm. Accessed December 16, 2012.

13. Chan DC, Tsou HH, Chen CY, Chen CY. Validation of the ChineseCanadian study of health and aging clinical frailty scale (CSHA-CFS) telephone version. Arch Gerontol Geriatr. 2010;50(3):e74-e80.

14. Hertzog MA. Considerations in determining sample size for pilot studies. Res Nurs Health. 2008;31(2):180-191.

15. Pang SM, Tse CY, Chan KS, et al. An empirical analysis of the decisionmaking of limiting life-sustaining treatment for patients with advanced chronic obstructive pulmonary disease in Hong Kong, China. $J$ Crit Care. 2004;19(3):135-144. 
16. Chan HYL, Pang SMC. Applicability of the modified Quality-ofLife Concerns in the End of Life Questionnaire (mQOLC-E) for frail older people. Asian Journal of Gerontology and Geriatrics. 2008;3(1):17-26.

17. Kim HY. Statistical notes for clinical researchers: assessing normal distribution (2) using skewness and kurtosis. Restor Dent Endod. 2013; 38(1):52-54.

18. Wong FKY, Chow S, Chung L, et al. Can home visits help reduce hospital readmissions? Randomized controlled trial. J Adv Nurs. 2008; 62(5):585-595.

19. Marek KD, Baker CD. Nurse home visit programs for the elderly. Annu Rev Nurs Res. 2006;24:157-178.

20. Elkan R, Kendrick D, Dewey M, et al. Effectiveness of home based support for older people: systematic review and meta-analysis. BMJ. 2001;323(7315):1-9.

21. Kwok T, Twinn S, Yan E. The attitudes of Chinese family caregivers of older people with dementia towards life sustaining treatment. $J A d v$ Nurs. 2007;58(3):256-262.
22. Desbiens NA, Mueller-Rizner N, Virnig B, Lynn J. Stress in caregivers of hospitalized oldest-old patients. J Gerontol A Biol Sci Med Sci. 2001; 56(4):M231-M235.

23. Lange MP. Family stress in the intensive care unit. Crit Care Med. 2001;29(10):2025-2026.

24. May J, Ellis-Hill C, Payne S. Gatekeeping and legitimization: how informal carers' relationship with health care workers is revealed in their everyday interactions. $J$ Adv Nurs. 2001;36(3):364-375.

25. Mor V, Besdine RW. Policy options to improve discharge planning and reduce rehospitalization. JAMA. 2011;305(3):302-303.

26. Holroyd EE. Chinese family obligation toward chronically ill elderly members: comparing caregivers in Beijing and Hong Kong. Qual Health Res. 2003;13(3):302-318.

27. Yam CH, Wong EL, Chan FW, Wong FY, Leung MC, Yeoh EK. Measuring and preventing potentially avoidable hospital readmissions: a review of the literature. Hong Kong Med J. 2010;16(5):383-389.
Clinical Interventions in Aging

\section{Publish your work in this journal}

Clinical Interventions in Aging is an international, peer-reviewed journal focusing on evidence-based reports on the value or lack thereof of treatments intended to prevent or delay the onset of maladaptive correlates of aging in human beings. This journal is indexed on PubMed Central, MedLine,

\section{Dovepress}

CAS, Scopus and the Elsevier Bibliographic databases. The manuscript management system is completely online and includes a very quick and fair peer-review system, which is all easy to use. Visit http://www.dovepress. com/testimonials.php to read real quotes from published authors. 